\title{
Dental Tissue as a Source of Stem Cells: Perspectives for Teeth Regeneration
}

Malgorzata Witkowska-Zimny

Department of Biophysics and Human Physiology, Medical University of Warsaw, Chalubinskiego 5, 02-004 Warsaw, Poland

\begin{abstract}
Stem cells are defined as cells that have the self-renew capacity and the ability to differentiate into one or more cell types. Recently, stem cell biology has become an interesting topic, especially because of the potential to use it in regenerative medicine. Several types of human stem cells have been isolated and identified in vivo and in vitro. This review highlights the characteristics and therapeutic potential of human dental progenitor/stem cells.
\end{abstract}

Keywords: Regenerative medicine; Stem cell; Dental tissue

Abbreviations: APDCs: Apical Papilla Derived Stem Cells; BMSCs: Bone Marrow Stromal/Stem Cells; DFPCs: Dental Follicle Progenitor Cells; DPSCs: Dental Pulp Stem Cells; PDLSCs: Periodontal Ligament Stem Cells; SHED: Stem Cells from Exfoliated Deciduous teeth; TEP: Tissue Engineered Product

\section{Introduction}

Tissue engineering is a relatively new branch of medicine that combines biology, engineering, clinical science to use it for reconstruction or generation new tissues and/or organs. Thus far, the developed strategies include the manipulation of the patient's own cells, the transplantation of stem cells, and the use of scaffold materials that trigger biological signals in order to accelerate the regenerative processes. The successful application of innovative therapies has been confirmed in clinical trials and experiments assessing the healing of broken bones, severe burns, blindness, deafness, heart, blood vessel, nerve and muscle damage, and in the treatment of many others diseases. In the nearest future cell-based therapy will represent a new strategy to treat a wide array of clinical conditions.

The term 'stem cells' may refer to various types of cells which (i) are unspecialized and can generate one or more cell lineages (ii) have the ability to replenish their own number (self- renewal). The types of stem cells are organized hierarchically from the most primitive through the targeted stem cells into mature tissue, and on the basis of this criterion the following stem cell classification has been proposed: totipotent, pluripotent, multipotent and unipotent stem cells. The most primitive stem cell is a totipotent zygote or first blastomers. They are able to give rise to the all embryonal tissues and also extra embryonic tissues i.e. the placenta. In the process of cell differentiation, they can gradually differentiate into pluripotent stem cells. During early embryogenesis, the cells are pluripotent and can give rise to all germ layers of the developing embryo: mesoderm, ectoderm and endoderm. The stem cells with lower potency are called "multipotent" stem cells and can differentiate into a number of cells, but only derived for the same germ layer. Multipotent stem cells give rise to unipotent stem cells that are committed to particular organs/tissues. They residue in specific niches that regulate their self-renewal, differentiation and are responsible for maintenance and regeneration of aged or damaged native tissue. Recent studies have revealed that adult/somatic stem cells may retain the potential to transdifferentiate from one phenotype to another, either in vitro or after transplantation in vivo. Stem/progenitor cells have been identified in most tissues of both developing and adult organism $[1,2]$. The dental tissues are no exception. A few studies gave evidence that teeth contain a special populations of adult stem cells. Although the regeneration potential of mammalian teeth is limited, the presence of stem cells was demonstrated in dental pulp, periodontal ligament, dental

papilla, and dental follicle, hence in the field of tooth engineering, many efforts have been made to explore this population of stem cells.

\section{Stem Cells in Dental Tissues}

Teeth develop from two interacting tissues: mesenchyme and ectodermal epithelium covering mouth with the original lining. The initiation of teeth formation starts around the 37th day of gestation. Teeth are complex organs containing dentine, enamel and dental follicle. The existence of stem cells within tooth was reported for the first time in 2000[3]. Shi group isolated cells with high proliferative potential for self-renewal from adult human dental pulp that are capable to develop into multiple cell lineages in vitro. So far five different human dental-tissue-derived stem/progenitor cells have been isolated and characterized (Figure 1). They can be classified in two main groups: first connected with dental pulp and second associated with the periodontium.

\section{Dental Pulp Stem Cells (DPSCs)}

DPSCs were the first cells isolated from dental pulp of third molars (wisdom teeth) from adults (19-29 years of age). The pulp tissue was gently separated from the crown and root and degradation using enzymatic digestion (collagenase and dispase) was performed. After filtration and washing in growth medium single-cell suspensions were seeded into culture plates. Thus isolated cells are able to adhere to plastic and form fibroblast-like colonies, extensively proliferate, form odontoblasts and possess the capacity to differentiate in vitro into various cell types e.g. osteoblast-, adipocytes-, neural-like cells under stimulation in cultured medium $[4,5]$. The best known example of an

*Corresponding author: Malgorzata Witkowska-Zimny, Medical University of Warsaw, Chalubinskiego 5, 02-004 Warsaw, Poland. Tel: +48 2262863 34; Fax: +48 2262878 46; E-mail: mwitkowska@wum.edu.pl

Received October 10, 2011; Accepted November 24, 2011; Published December 12, 2011

Citation: Witkowska-Zimny M (2011) Dental Tissue as a Source of Stem Cells: Perspectives for Teeth Regeneration. J Bioengineer \& Biomedical Sci S2:006 doi:10.4172/2155-9538.S2-006

Copyright: (C) 2011 Witkowska-Zimny M. This is an open-access article distributed under the terms of the Creative Commons Attribution License, which permits unrestricted use, distribution, and reproduction in any medium, provided the original author and source are credited. 


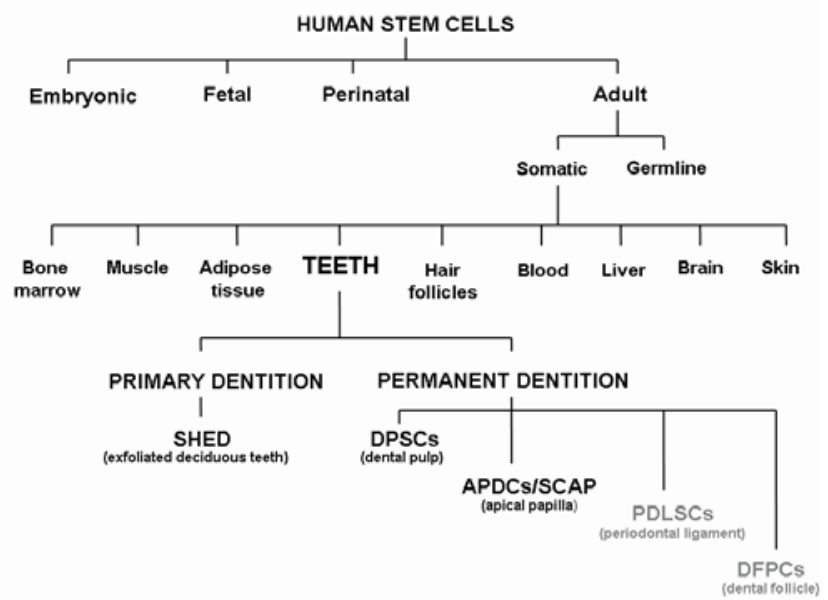

Figure 1: Diagram of human stem cells. Dental stem/progenitor cells associated with periodontium are colored in grey.

adult stem cell is bone marrow stromal/stem cell (BMSCs), considered to be the gold standard in the assessment of in vitro differentiation of mesenchymal stem cells. Newly discovered type of progenitor and stem cells are compared with them. DPSCs occurred at an apparently higher frequency in comparison to the BMSCs. The proliferation analysis revealed that human DPSCs have faster population doubling time than BMSCs in vitro. Shi group $[3,6]$ studies demonstrated that DPSCs and BMSCs share a similar pattern of protein expression. DPSCs can be long-term cultured with no sign of senescence or changes in viability, phenotype, and genotype [7]. However, it was demonstrated DPSCs telomere length decreases during extensive cell proliferation. In eukaryotes, the chronological cell ageing is studied through telomere shortening of their chromosomes. It is not clear if this observation can diminish transplantation capacity of these stem cells in therapeutic applications.

After post transplantation into immunocompromised mice, DPSCs generated a dentine/pulp-like structure in vivo and expressed humanspecific transcripts for dentin matrix component like osteocalcin or bone sialoprotein. It is interesting that DPSCs exist and can be isolated also from inflamed pulps, but there are some differences between DPSCs derived from inflamed and normal pulps [8].

Access to the DPSCs collection site is relatively easy. As they can be extracted with high efficiency, exert extensive differentiation ability, and demonstrate interactivity with biomaterials what DPSCs are considered ideal for tissue reconstruction.

\section{Apical Papilla Derived Stem Cells (APDCs, also stem cells from apical papilla/SCAP)}

Mesenchymal cells within the dental papilla are responsible for production of dentin and pulp. First report about new class of cells with high proliferation activity and multilineage differentiation potential obtained from immature tip of the apical papilla of human developing third molars was published by Abe et al. $[9,10]$. Since then, only few information on human progenitor cells from apical papilla is available in the literature. The capacity of APDCs to differentiate into functional dentinogenic cells has been verified using animal models. They are capable to form odontoblast-like cells and produce dentin in vivo and can serve as a cell source of primary odontoblasts for root dentin formation [11]. They are only weakly immunogenic and inhibit the proliferation of $\mathrm{T}$ cells and a one-way mixed lymphocyte reaction (MLR) in vitro. The mechanism of this immunomodulatory property and its function in vivo is unknown [12].

\section{Periodontal Ligament Stem Cells (PDLSCs)}

The mesenchyme of the dental sac condenses to form the periodontal ligament (fiber). Periodontal ligemant (PDL) is a tissue that connects cementum and alveolar bone to maintain and support teeth in situ. PDL perform supportive, sensory, nutritive, regenerative and homeostatic functions. After separation of PDL from the surface of the third molar root and enzymatic digestion, a unique stem-cell population can be isolated. These cells are plastic-adherent when maintained under standard culture conditions and have the ability to differentiate into cementoblast-like cells, adipocytes in vitro, and cementum/PDL-like tissue in vivo [13]. It is considered that PDLSCs have a potential for periodontal tissues regeneration. It must be mentioned that periodontal diseases are the main cause of tooth loss and are a substantial public health burden worldwide.

\section{Dental Follicle Progenitor Cells (DFPCs)}

Dental follicle consists of tooth germline tissues. Ectomesenchyme surrounds the dental/enamel organ and form dental follicle which plays an important role in tooth development and will produce all the supporting structure of a tooth: cementum, alveolar bone, and periodontal ligaments. Dental follicle cells can be easily isolated after wisdom tooth extraction. Third molar is very often extracted during orthodontic therapy or to avoid inflammation, so dental follicle, like other parts of third molars, is commonly discarded as medical waste. Thus third molars could be a practical cells source for potential therapeutic applications. The existence of progenitor/stem cells within dental follicle was reported for the first time in 2002 [14]. DFPCs can differentiate toward a cementoblast, osteoblast, periodontal ligament, adipogenic, osteogenic and neuronal lineage $[15,16]$. Tsuchiya et al. [17] results suggest that DFSCs potential for bone formation is similar to BMSCs. Like PDLSCs which originate from DFPCs, they are also a promising tool for periodontal tissue regeneration.

\section{Stem Cells from Exfoliated Deciduous Teeth (SHED)}

At about 7 years of age the process of replacement of 20 deciduous teeth for 32 permanent teeth begins. Unexpectedly, Miura et al. [18] isolated a distinctive population of multipotent stem cells from the remnant pulp of exfoliated deciduous teeth. The method of isolation was the same as for DPSCs. Stem cells from human exfoliated deciduous teeth (SHED) are highly proliferative population of clonogenic cells capable of differentiating into a variety of cell types including neural cells, adipocytes, and odontoblasts. It has been shown that SHED have the highest proliferation rates in vitro compared to BMSCs or even DPSCs [19]. SHED transplanted on scaffolds/tooth sliced into immunodeficient mice differentiated into functional odontoblasts and generated dentine and angiogenic endothelium [20]. Because children naturally lose deciduous teeth, there are multiple opportunities to harvest this type of stem/progenitor cells in painless and minimally invasive procedure. Thus exfoliated teeth can be a unique resource for stem-cell transplantation in regenerative dentistry. Besides, in the future, the possibility to bank these cells may provide sources both for allogenic and autologous cell replacement in later life.

\section{Perspectives}

Technologies using stem/progenitor cells might be the new era 
of personalized medicine. There is no doubt that the description of tissue engineery offers a new hope to both patient who suffer with tooth loss and the dentist as well. Although teeth are nonessential for life and thus they are not considered a major target for regenerative medicine research like for example bone or blood vessels; however, they are extremely desirable by patient mainly for aesthetic reasons. Dental stem/progenitor cells could be feasible tools for dental tissue engineering. Numerous attempts have been made to produce a tooth and very promising results have been made. There are a few approaches which are investigated in reparative and reconstructive dentistry. The first concerns the placing of stem cell into the scaffold material and subsequent implantation of the construct elsewhere in the body. The tooth will be grown and matured, and finally extracted and implanted into the oral cavity [21]. In another, stem cells released from the tooth tissue are implanted into a severely injured tooth to help to regenerate the pulp and restore the tooth/teeth. Huang et al. [22] obtained vascularized human dental pulp in emptied root canal space and new dentin on existing dentinal walls using human stem/progenitor cells in immunocompromised mouse model.

There is a growing number of preclinical studies examining the potential of dental-tissue- derived stem/progenitor cells. They have been used in tissue engineering studies in animals: (i) Sonoyama et al. [23] using a minipig model, transplanted both human SCAP and periodontal ligament stem cells (PDLSCs) to generate a root/ periodontal complex capable of supporting a porcelain crown, resulting in normal tooth function; (ii) Liu et al. [24] explored the potential of using autologous periodontal ligament stem cells (PDLSCs) to treat periodontal defects in a porcine model of periodontitis.

Tissue engineered products (TEPs) consisting of scaffold and cells will become an alternative to the currently used methods of substitution and regeneration of damaged or removed tissues. Despite the hope evoked by regenerative dentistry, patients and dentists eagerly anticipate TEPs. Hence, we still need a lot of patience until TEPs become easily accesible on the medical market. There is no product approved in reparative and reconstructive dentistry, but there are some extensively studied on the way. It is certain that the field has the explosive potency.

Based on the promising results of animal experiments with dental stem/progenitor cells, a few clinical trials have also been initiated. However, compared with the more conventional sources of stem cells i.e. bone marrow or adipose tissue, the number of reports describing the results of clinical investigations using stem cells derived from dental tissues is limited. The ongoing human clinical trials using stem/ progenitor cells isolated from dental tissues are summarized in Table 1.

Since the discovery of the dental pulp stem cells about 10 years ago, several studies have reported various types of stem/progenitor cells in mature permanent teeth, developing teeth, and tooth germs [3,25-27].

However, to date, there are few published papers concerning their

\begin{tabular}{|c|l|l|l|}
\hline $\begin{array}{c}\text { Cells } \\
\text { sources }\end{array}$ & \multicolumn{1}{|c|}{ Indication (Title) } & Phase & \multicolumn{1}{|c|}{ Country } \\
\hline $\begin{array}{l}\text { Periodontal } \\
\text { ligament }\end{array}$ & $\begin{array}{l}\text { Periodontal Tissue Regeneration } \\
\text { Using }\end{array}$ & I & China \\
\cline { 2 - 4 } & $\begin{array}{l}\text { Periodontal Ligament Stem Cell } \\
\text { Implantation in the Treatment of Peri- } \\
\text { odontitis }\end{array}$ & I, II & China \\
\hline $\begin{array}{l}\text { Effect of Cyclic Tensional Force } \\
\text { on Osteogenic Differentiation of Human } \\
\text { Periodontal Ligament Stem Cells }\end{array}$ & $\begin{array}{l}\text { No } \\
\text { information } \\
\text { available }\end{array}$ & Taiwan \\
\hline
\end{tabular}

Table1: Current status of dental tissues stem/progenitor cells in clinical trials (based on www.clinicaltrials.gov). in-depth characterization. Despite the fact that dental-tissue-derived stem/progenitor cells share several common characteristics (high proliferation rate, multidifferentiation potential, high viability), they present significant heterogeneity, what may be enhanced with their different function in tissue microenvironment.

In 2006, the Mesenchymal and Tissue Stem Cell Committee of the International Society for Cellular Therapy proposed a minimal set of four criteria for the identification of human mesenchymal stem cells [28], namely: (i) they have to be plastic-adherent when maintained under standard culture conditions; (ii) they must have the ability for osteogenic, adipogenic, and chondrogenic differentiation; (iii) they must express CD73, CD90, CD105; and (iv) they must lack the expression of hematopoietic linage markers (CD14, CD11b, CD34, CD45, CD19, CD79). Only CD73 and Stro- 1 surface protein expression were confirmed in all dental precursor cells, but many studies are incomplete and does not include a thorough characterization of the surface markers. So at the moment, calling these cells as "stem cells" may be a slight exaggeration. Deeper knowledge of stem cells markers, appropriate validation of cell type, optimal performance, and further characterization of these progenitor cells from dental tissues should yield a demonstrable benefit in cell therapy.

There is little information about the immunological features of dental-related stem/progenitor cells in the literature. They are available only in specific points in life, some of them only during wisdom tooth eruption. Their quantities are debatable. Ideally, stem cells for regenerative medical applications should be found in abundant quantities, harvestable in a minimally invasive procedure, then safely and effectively transplanted to either an autologus or allogenic host. Today, the practical use of dental stem/progenitor cells is still problematic.

Without fail, the dental stem cells biology might provide meaningful insights into the development of dental tissue and cellular differentiation processes. There are also some safety issues inherent in stem cell therapy. Like any other new technology, it is completely unknown what the long-term effects of such interference with nature could be. Before the development of effective cellular-based therapies it is necessary to better understand the mechanisms of self- renewal (allow us to regulate stem cells growth, generate sufficient cell number and decreased risk of unlimited malignant transformation), regulation of differentiation and specific tissue production, and interaction stem cells with immune system.

\section{Conclusions}

Tissue engineering is defined as "an interdisciplinary field that applies the principles of engineering and the life sciences toward the development of biological substitutes that restore, maintain, or improve tissue function or a whole organ." [29]. There are several potential aspects of using cells in reparative and reconstructive of dental tissues. To date, five different human dental stem/progenitor cells have been isolated. Stem cells based tissue engineering is thought to be a promising way to replace the missing tooth and regenerate damaged tooth structures. The successful use of all types of stem cells in regeneration therapy may be achieved only after understanding of the basic molecular mechanisms underlying cell fate switching of stem cells. It will be an essential contribution to ensure their safe use in regenerative medicine. 
Citation: Witkowska-Zimny M (2011) Dental Tissue as a Source of Stem Cells: Perspectives for Teeth Regeneration. J Bioengineer \& Biomedical Sci S2:006. doi:10.4172/2155-9538.S2-006

\section{Acknowledgments}

Research in the author's laboratory is supported by project no. N N302157037 from the Polish funds for scientific research in 2009-2012.

\section{References}

1. Witkowska-Zimny M, Walenko K (2011) Stem cells from adipose tissue. Cell Mol Biol Lett 16: 236-257.

2. Witkowska-Zimny M, Wrobel E (2011) Perinatal sources of mesenchymal stem cells: Wharton's jelly, amnion and chorion. Cell Mol Biol Lett16: 493-514.

3. Gronthos S, Mankani M, Brahim J, Robey PG, Shi S (2000) Postnatal human dental pulp stem cells (DPSCs) in vitro and in vivo. Proc Natl Acad Sci U S A 97: 13625-13630.

4. Gronthos S, Brahim J, Li W, Fisher LW, Cherman N, et al. (2002) Stem cell properties of human dental pulp stem cells. J Dent Res 81: 531-535.

5. Morsczeck C (2006) Gene expression of runx2, Osterix, c-fos, DLX-3, DLX-5, and MSX-2 in dental follicle cells during osteogenic differentiation in vitro. Calcif Tissue Int 78: 98-102.

6. Liu H, Gronthos S, Shi S (2006) Dental pulp stem cells. Methods Enzymol 419: 99-113.

7. Mokry J, Soukup T, Micuda S, Karbanova J, Visek B, et al. (2010) Telomere attrition occurs during ex vivo expansion of human dental pulp stem cells. $J$ Biomed Biotechnol 2010: 673513.

8. Alongi DJ, Yamaza T, Song Y, Fouad AF, Romberg EE, et al. (2010) Stem/ progenitor cells from inflamed human dental pulp retain tissue regeneration potential. Regen Med 5: 617-631.

9. Abe S, Yamaguchi S, Watanabe A, Hamada K, Amagasa T (2008) Hard tissue regeneration capacity of apical pulp derived cells (APDCs) from human tooth with immature apex. Biochem Biophys Res Commun 371: 90-93.

10. Abe S, YamaguchiS, Amagasa T (2007) Multilineage cells from apical pulp of human tooth with immature apex. Oral Science International 4: 45-58.

11. Sonoyama W, Liu Y, Yamaza T, Tuan RS, Wang S, et al. (2008) Characterization of the apical papilla and its residing stem cells from human immature permanent teeth: a pilot study. J Endod 34: 166-171.

12. Ding G, Liu Y, An Y, Zhang C, Shi S, et al. (2010) Suppression of T cell proliferation by root apical papilla stem cells in vitro. Cells Tissues Organs 191 357-364.

13. Seo BM, Miura M, Gronthos S, Bartold PM, Batouli S, et al. (2004) Investigation of multipotent postnatal stem cells from human periodontal ligament. Lancet 364: 149-155.

14. Handa K, Saito M, Yamauchi M, Kiyono T, Sato S et al. (2002) Cementum matrix formation in vivo by cultured dental follicle cells. Bone 31: 606-611.

15. Morsczeck C, Götz W, Schierholz J, Zeilhofer F, Kühn U, et al. (2005) Isolation of precursor cells (PCs) from human dental follicle of wisdom teeth. Matrix Bio 24: $155-165$

16. Yao S, Pan F, Prpic V, Wise GE (2008) Differentiation of stem cells in the denta follicle. J Dent Res 87: 767-771.

17. Tsuchiya S, Ohshima S, Yamakoshi Y, Simmer JP, Honda MJ (2010) Osteogenic differentiation capacity of porcine dental follicle progenitor cells. Connect Tissue Res 51: 197-207.

18. Miura M, Gronthos S, Zhao M, Lu B, Fisher LW, et al. (2003) SHED: stem cells from human exfoliated deciduous teeth. Proc Nat IAcad Sci US A 100 5807-5812.

19. Nakamura S, Yamada Y, Katagiri W, Sugito T, Ito K, et al. (2009) Stem cell proliferation pathways comparison between human exfoliated deciduous teeth and dental pulp stem cells by gene expression profile from promising dental pulp. J Endod 35: 1536-1542.

20. Sakai VT, Zhang Z, Dong Z, Neiva KG, Machado MA, et al. (2010) SHED differentiate into functional odontoblasts and endothelium. J Dent Res 89: 791 796.

21. Zhang W, Walboomers XF, van Kuppevelt TH, Daamen WF, Bian Z, et al. (2006) The performance of human dental pulp stem cells on different threedimensional scaffold materials. Biomaterials 27: 5658-5668.

22. Huang GT, Yamaza T, Shea LD, Djouad F, Kuhn NZ, et al. (2010) Stem/ progenitor cell-mediated de novo regeneration of dental pulp with newly deposited continuous layer of dentin in an in vivo model. Tissue Eng Part $A$ 16: $605-615$

23. Sonoyama W, Liu Y, Fang D, Yamaza T, Seo BM, et al. (2006) Mesenchyma stemcell- mediated functional tooth regeneration in swine. PLoS One 1: e79.

24. Liu Y, Zheng Y, Ding G, Fang D, Zhang C , et al.(2008) Periodontal ligament stem cell- mediated treatment for periodontitis in miniature swine. Stem Cells 26: 1065-1073.

25. Huang GT, Gronthos S, Shi S (2009) Mesenchymal stem cells derived from dental tissues vs. those from other sources: their biology and role in regenerative medicine. J Dent Res 88: 792-806.

26. Nishino Y, Yamada Y, Ebisawa K, Nakamura S, Okabe K, et al. (2011) Stem cells from human exfoliated deciduous teeth (SHED) enhance wound healing and the possibility of novel cell therapy. Cytotherapy 13: 598-605.

27. Ikeda E, Yagi K, Kojima M, Yagyuu T, Ohshima A, et al. (2008) Multipotent cells from the human third molar: feasibility of cell-based therapy for liver disease. Differentiation 76: 495-505.

28. Dominici M, Le Blanc K, Mueller I, Slaper-Cortenbach I, Marini F, et al. (2006) Minimal criteria for defining multipotent mesenchymal stromal cells. The International Society for Cellular Therapy position statement. Cytotherapy 8 : 315-317.

29. Langer R, Vacanti JP (1993) Tissue engineering. Science 260: 920-926.
This article was originally published in a special issue, Regenerative Medicine handled by Editor(s). Dr. Lisa M Larkin, University of Michigan, USA. 